\title{
Positive Correlation between Severity of Blepharospasm and Thalamic Glucose Metabolism
}

\author{
Hideki Muraia, ${ }^{a}$ Yukihisa Suzukia, ${ }^{a}$ Motohiro Kiyosawa ${ }^{a}$ \\ Masato Wakakurac Manabu Mochizuki ${ }^{\mathrm{a}}$ Kiichi Ishiwata $^{\mathrm{b}}$ \\ Kenji Ishiib \\ ${ }^{a}$ Department of Ophthalmology and Visual Science, Tokyo Medical and Dental \\ University, Graduate School, bPositron Medical Center, Tokyo Metropolitan \\ Institute of Gerontology, and Inouye Eye Hospital, Tokyo, Japan
}

\section{Key Words}

Blepharospasm · Cerebral glucose metabolism · Dystonia $\cdot$ Positron-emission tomography · Thalamus

\begin{abstract}
A 43-year-old woman with drug-related blepharospasm was followed up for 22 months. She had undergone etizolam treatment for 19 years for indefinite complaints. We examined her cerebral glucose metabolism 5 times (between days 149 and 688 since presentation), using positron emission tomography, and identified regions of interest in the thalamus, caudate nucleus, putamen, and primary somatosensory area on both sides. The severity of the blepharospasm was evaluated by PET scanning using the Wakakura classification. Sixteen women (mean age $42.4 \pm 11.7$ years) were examined as normal controls. The thalamic glucose metabolism in our patient was significantly increased on days 149,212 , and 688 . The severity of the blepharospasm was positively correlated with the thalamic glucose metabolism, suggesting that the severity of blepharospasms reflects thalamic activity.
\end{abstract}

\section{Introduction}

Essential blepharospasm (EB) is a form of focal dystonia characterized by involuntary movement of the musculature in the upper face. Several researchers have studied focal dystonia using positron emission tomography (PET) and ${ }^{18} \mathrm{~F}$-fluorodeoxyglucose $\left({ }^{18} \mathrm{~F}\right.$ FDG). Galardi et al. [1] reported hypermetabolism in the thalamus, basal ganglia, anterior cingulate gyrus, and cerebellum in patients with spasmodic torticollis, and EsmaeliGutstein et al. [2] found a relative increase in metabolic activity in the thalamus of patients with EB. Suzuki et al. [3] have reported glucose hypermetabolism in the thalamus 
of patients with EB and suggested that dysfunction of the basal ganglia-thalamocortical motor circuit may play a major role in the pathophysiology of dystonia.

We hypothesized that the severity of blepharospasm is correlated to the activity in the thalamus or basal ganglia. In the present study, a patient with drug-induced blepharospasm was followed up and her cerebral glucose metabolism was recorded 5 times using PET and ${ }^{18} \mathrm{~F}$-FDG. We assume that the pathophysiologies of drug-related blepharospasm and EB are similar. Glucose metabolism in the thalamus, the basal ganglia, and the primary somatosensory area (PSA) were evaluated and the relationship between the severity of blepharospasm and glucose metabolism in these areas was analyzed.

\section{Materials and Methods}

A 43-year-old woman presented with difficulty in opening her eyes (day 0 ). She had no organic brain disorder or other neuropsychiatric disease as evaluated by neurologists using conventional diagnostic magnetic resonance imaging (MRI) and no family history of dystonic diseases. She had taken $3 \mathrm{mg} /$ day of etizolam for 19 years for the treatment of indefinite complaints, and the blepharospasm had developed recently [4]. She received an injection of botulinum-A toxin administered bilaterally to the orbicularis oculi on day 72, with unsatisfactory results. However, upon discontinuation of etizolam, her symptoms gradually improved in the course of a year. PET scanning was performed 5 times in the course of 22 months (on days 149, 212, 310,443, and 688), and the severity of the spasms at the time of PET scanning was evaluated according to the Wakakura classification [5].

Sixteen women (mean age, $42.4 \pm 11.7$ years) were recruited as normal controls, and MRI and ${ }^{18} \mathrm{~F}-$ FDG PET were performed. Informed consent was obtained from all study participants. The protocol of this study was approved by the institutional ethics committee of the Tokyo Metropolitan Institute of Gerontology. All the procedures conformed to the tenets of the Declaration of Helsinki.

MR images were obtained with a 1.5-Tesla Signa Horizon scanner (General Electric, Milwaukee, Wisc., USA) in order to register PET and MR images. Transaxial images with T1-weighted contrast [3D-SPGR, repetition time $(\mathrm{TR})=9.2 \mathrm{~ms}$, echo time $(\mathrm{TE})=2.0 \mathrm{~ms}$, matrix size $=256 \times 256 \times 124$, voxel size $=0.94 \times 0.94 \times 1.3 \mathrm{~mm}$ ] and T2-weighted contrast (first spin echo, TR $=3,000 \mathrm{~ms}, \mathrm{TE}=100 \mathrm{~ms}$, matrix size $=256 \times 256 \times 20$, voxel size $=0.7 \times 0.7 \times 6.5 \mathrm{~mm}$ ) were obtained.

PET scans were obtained using the SET 2400W whole-body scanner (Shimadzu, Kyoto, Japan) at the Positron Medical Center, Tokyo Metropolitan Institute of Gerontology. Attenuation was corrected by a transmission scan with a ${ }^{68} \mathrm{Ga} /{ }^{68} \mathrm{Ge}$ rotating source. A bolus of $150 \mathrm{MBq}{ }^{18} \mathrm{~F}-\mathrm{FDG}$ was injected intravenously. The subjects were then requested to lie down comfortably with their eyes closed. A 6-min emission scan in the 3D acquisition mode was started $45 \mathrm{~min}$ after the injection, and 50 transaxial images with an interslice interval of $3.125 \mathrm{~mm}$ were obtained. The tomographic images were reconstructed using a filtered backprojection method with a Butterworth filter (cutoff frequency: 1.25 cycles/cm and order of 2).

PET images were three-dimensionally registered to their 3D-SPGR MR images using the Automated Medical Images Registration (AMIR) program [6]. Further data analysis was performed on LINUX workstations using the Dr. View program (AJS, Tokyo, Japan). Regions of interest (ROIs), i.e. 8.5-mmdiameter circles, were defined interactively on the resliced PET images by visual inspection with reference to the corresponding MR image. The ROIs were placed over the bilateral thalamus, caudate, putamen, and PSA, and the cerebral glucose metabolism in each region was measured. The values of cerebral glucose metabolism in the ROIs were normalized according to the global cerebral glucose metabolism, which was calculated by averaging voxels $>20 \%$ of the maximum value. The normalized values of glucose metabolism in each area in the patient were compared to the corresponding average values in the normal controls, and a difference of $2.5 \mathrm{SD}$ from the controls was considered statistically significant. The correlation coefficient between the severity of blepharospasm and the normalized glucose metabolism in each area studied was also calculated. 


\section{Results}

The glucose metabolism in the thalamus of the patient was increased significantly on days 149 and 212 (table 1). However, a significant change was not observed in the other areas (caudate, putamen, and PSA). The severity of the blepharospasm was 3 on day 149, 2 on day 212,1 on day 310,1 on day 443 , and 2 on day 688 . Regression analyses with the data of the bilateral thalamus demonstrated a significant positive correlation between cerebral glucose metabolism and the severity of the blepharospasm [correlation coefficient (r): right: 0.853 ( $<$ 0.05), left: 0.953 ( $\mathrm{p}<0.01$ )] (

\section{Discussion}

We observed cerebral glucose hypermetabolism in the thalamus of a patient with drugrelated blepharospasm. Reduced cerebral glucose metabolism in the thalamus was found to be correlated with an improvement in the blepharospasm. Thus, the activity in the thalamus may be related to the severity of blepharospasms.

Blepharospasm is a dystonic disorder, and there are several reports on cerebral glucose hypermetabolism in the thalamus of patients with dystonia [1-3, 7], including our previous report. Esmaeli-Gutstein et al. [2] classified blepharospasms on the basis of severity (mild, moderate, and severe) in 10 patients and examined the relationship between the severity and cerebral glucose metabolism in the thalamus using ${ }^{18} \mathrm{~F}-\mathrm{FDG}$ PET. They did not find a correlation between glucose metabolism and blepharospasm severity in their study, possibly because the interval between the most recent botulinum toxin injection and PET was not uniform in each patient.

Further, Odergren et al. [7] measured cerebral blood flow in four patients with writer's cramp and four controls, using PET. They reported that the perceived difficulty and signs of dystonia progressed with the duration of writing, which in turn correlated with increased activity in the thalamus of the patients. Many studies have reported thalamic hyperactivity in patients with dystonic diseases, but the neurophysiological mechanisms of this hyperactivity are not well defined. In our previous study, we observed glucose hypermetabolism in the thalamus of EB patients whose spasms were suppressed by botulinum toxin treatment [3]. This result may indicate that the hyperactivity in the thalamus is caused by the blepharospasm itself.

On the other hand, the hyperactivity in the thalamus may be secondary to dystonic muscle spasms. There are 2 possible explanations for the hyperactivity in the thalamus. First, the somatosensory input from the skin induced by dystonic movement may activate the basal ganglia-thalamocortical motor circuit. Gobbelé et al. [8] investigated the interference of tactile and painful stimuli on thalamocortical processing of somatosensory information in humans, using somatosensory evoked potentials. They observed that the thalamic source was significantly increased during pain interference. Second, the activation of the thalamus may be due to neuronal projection from the muscle spindle that contracts with dystonic muscle spasms. Katter et al. [9] observed that the neurons that project from the jaw-muscle spindle to the thalamus were activated with microelectrodes in the rat. In the present study, we observed a patient who developed blepharospasm after long-term etizolam treatment. Wakakura et al. [4] examined 254 patients with blepharospasm and reported that $13.8 \%$ of them had used etizolam before 
the onset of blepharospasm. They concluded that prolonged use of benzodiazepines or thienodiazepines may induce blepharospasm. The clinical features of drug-related blepharospasm are similar to those of $\mathrm{EB}$, and the spasms may resolve on discontinuing the use of related drugs [4]. In our case, the symptoms of blepharospasm improved to some extent after etizolam was discontinued and, thus, the patient's condition could be diagnosed as drug-related blepharospasm.

\section{Acknowledgements}

The authors have no conflict of interest. However, this research was supported by Grants-in-Aid for Scientific Research (B) 21791725 (Y. Suzuki) and (C) 20591038 (K. Ishii) from the Japan Society for the Promotion of Science.

Table 1. Cerebral glucose metabolism in a patient with blepharospasm

\begin{tabular}{|c|c|c|c|c|c|c|}
\hline \multirow{2}{*}{ Day } & \multirow[t]{2}{*}{ Severity } & \multirow[t]{2}{*}{$\mathrm{R} / \mathrm{L}$} & \multicolumn{4}{|l|}{ Brain area } \\
\hline & & & thalamus & caudate & putamen & PSA \\
\hline \multirow[t]{2}{*}{149} & 3 & $\mathrm{R}$ & $1.64^{*}$ & 1.28 & 1.46 & 1.20 \\
\hline & & $\mathrm{L}$ & $1.66^{*}$ & 1.24 & 1.44 & 1.15 \\
\hline \multirow[t]{2}{*}{212} & 2 & $\mathrm{R}$ & $1.57^{*}$ & 1.39 & 1.46 & 1.18 \\
\hline & & $\mathrm{L}$ & $1.63^{*}$ & 1.43 & 1.47 & 1.12 \\
\hline \multirow[t]{2}{*}{310} & 1 & $\mathrm{R}$ & 1.52 & 1.45 & 1.53 & 1.23 \\
\hline & & $\mathrm{L}$ & 1.51 & 1.33 & 1.39 & 1.25 \\
\hline \multirow[t]{2}{*}{443} & 1 & $\mathrm{R}$ & 1.49 & 1.31 & 1.44 & 1.13 \\
\hline & & $\mathrm{L}$ & 1.52 & 1.45 & 1.42 & 1.14 \\
\hline \multirow[t]{2}{*}{688} & 2 & $\mathrm{R}$ & 1.52 & 1.41 & 1.48 & 1.17 \\
\hline & & $\mathrm{L}$ & 1.56 & 1.43 & 1.44 & 1.18 \\
\hline \multirow{2}{*}{\multicolumn{2}{|c|}{ Normal average }} & $\mathrm{R}$ & $1.37 \pm 0.07$ & $1.41 \pm 0.08$ & $1.51 \pm 0.14$ & $1.15 \pm 0.07$ \\
\hline & & $\mathrm{L}$ & $1.37 \pm 0.08$ & $1.41 \pm 0.09$ & $1.51 \pm 0.12$ & $1.15 \pm 0.06$ \\
\hline
\end{tabular}

Values indicate glucose metabolism in the ROIs, as normalized according to the global cerebral glucose metabolism.

* Significant increase (>normal average $+2.5 \mathrm{SD}$ ). 


\begin{tabular}{l|l|l|l} 
Case Reports in & $\begin{array}{l}\text { Case Rep Ophthalmol 2011;2:50-54 } \\
\text { DOI: } 10.1159 / 000324459\end{array}$ & $\begin{array}{l}\text { Published online: } \\
\text { February 5, 2011 }\end{array}$ & $\begin{array}{l}\text { ISSN 1663-2699 } \\
\text { ISww.karger.com/cop }\end{array}$ \\
\hline
\end{tabular}

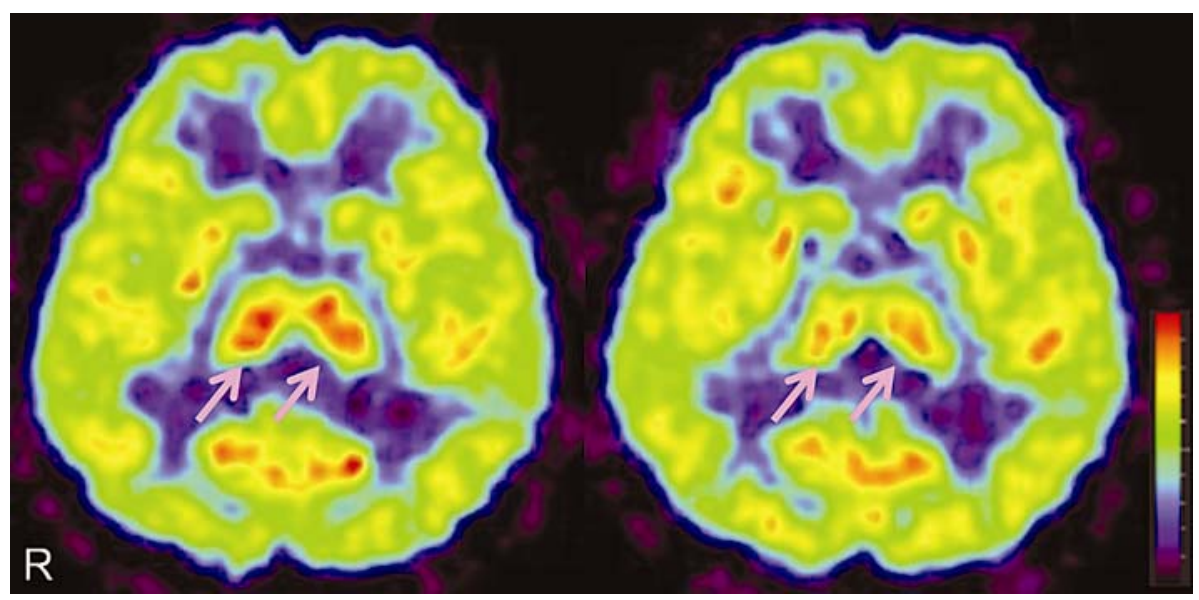

Fig. 1. Images of PET with ${ }^{18} \mathrm{~F}-\mathrm{FDG}$ in a patient with blepharospasm on days 149 (left) and 443 (right) after presentation. The severity of the blepharospasm was 3 on day 149 and 1 on day 443 . The glucose metabolism in the thalamus was high on day 149 and low on day 443 (arrows).

\section{References}

1 Galardi G, Perani D, Grassi F, Bressi S, Amadio S, Antoni M, Comi GC, Canal N, Fazio F: Basal ganglia and thalamo-cortical hypermetabolism in patients with spasmodic torticollis. Acta Neurol Scand 1996;94:172-176.

$\checkmark 2$ Esmaeli-Gutstein B, Nahmias C, Thompson M, Kazdan M, Harvey J: Positron emission tomography in patients with benign essential blepharospasm. Ophthal Plast Reconstr Surg 1999;15:23-27.

3 Suzuki Y, Mizoguchi S, Kiyosawa M, Mochizuki M, Ishiwata K, Wakakura M, Ishii K: Glucose hypermetabolism in the thalamus of patients with essential blepharospasm. J Neurol 2007;254:890-896.

$\checkmark 4$ Wakakura M, Tsubouchi T, Inouye J: Etizolam and benzodiazepine induced blepharospasm. J Neurol Neurosurg Psychiatry 2004;75:506-509.

5 Wakakura M: [Blepharospasm and hemifacial spasm]. Nippon Ganka Gakkai Zasshi 2005;109:667-680 (in Japanese).

6 Ardekani BA, Braun M, Hutton BF, Kanno I, Iida H: A fully automatic multimodality image registration algorithm. J Comput Assist Tomogr 1995;19:615-623.

7 Odergren T, Stone-Elander S, Ingvar M: Cerebral and cerebellar activation in correlation to the action-induced dystonia in writer's cramp. Mov Disord 1998;13:497-508

$\checkmark 8$ Gobbelé R, Halboni P, Buchner H, Waberski TD: Interference of tactile and pain stimuli on thalamocortical signal processing in humans revealed by median nerve SEPs. Clin Neurophysiol 2007;118:2497-2505.

-9 Katter JT, Dado RJ, Kostarczyk E, Giesler GJ Jr: Spinothalamic and spinohypothalamic tract neurons in the sacral spinal cord of rats. I. Locations of antidromically identified axons in the cervical cord and diencephalon. J Neurophysiol 1996;75:2581-2605. 